\title{
Improved Endurance Gain in BALB / C Mice by a Nanotechnology Medical Device
}

\author{
Silvia Altobello ${ }^{1}$, Antonio Filippone ${ }^{2}$, Luigi Grillo ${ }^{3}$, De Martino Angelo ${ }^{4}$, Beninati Simone ${ }^{4}$, \\ Alessandro Pumpo 5
}

${ }^{1}$ Department of General Medicine, Haybusak University, Yerevan, Republic of Armenia

${ }^{2}$ Department of Biomedical and Biotechnology, University of Catania, Catania, Italy

${ }^{3}$ Scientific Institutes of Hospitalization and Care (IRCCS), Rome, Italy

${ }^{4}$ Department of Biology, University of Tor Vergata, Rome, Italy

${ }^{5}$ Department of Clinical Biochemistry, University S. Raffaele, Rome, Italy

Email address:

beninati@bio.uniroma2.it (B. Simone)

\section{To cite this article:}

Silvia Altobello, Antonio Filippone, Luigi Grillo, De Martino Angelo, Beninati Simone, Alessandro Pumpo. Improved Endurance Gain in BALB / C Mice by a Nanotechnology Medical Device. International Journal of Biomedical Materials Research. Vol. 6, No. 1, 2018, pp. 13-19. doi: 10.11648/j.ijbmr.20180601.13

Received: February 1, 2018; Accepted: February 24, 2018; Published: March 22, 2018

\begin{abstract}
The main finding of the present study was the improved endurance gain in BALB / C mice, following the

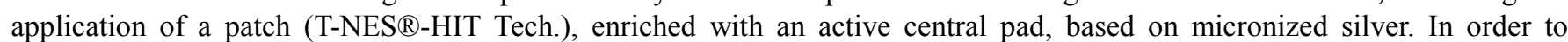
transfer very thin Extremely Low Frequency (ELF) magnetoelectric signals, the pad contains a nanotechnological material (silver micronized by plasma vaporization) that is appropriately activated with optical, magnetic, electrical, and acoustic signals. It has been reported previously that the ELF magnetic field, induces an action on cellular glycocalics, fundamental for cellular communication and recognition, and a direct activation of protons and ionic fluxes. The treated animals underwent exercises with Rotarod and several parameters were evaluated and compared with untreated control mice. The following parameters were measured: steady state generic training, long slow distance, high intensity "endurance" and steady state split session training. The ELF magnetic field of T-NES®-HIT Tech. micronized silver patch device, applied to BALB / C mice, markedly improved their endurance gain performance as evidenced by the increase of their aerobic capacity, maximum heart rate and maximum aerobic speed.
\end{abstract}

Keywords: Endurance, ELF Magnetic Field, Rotarod Test

\section{Introduction}

The induction of a cellular effect by a nanotechnologic device has as its premise the ability to guide the various metabolic phases through mechanisms that act on all interfering phenomena, and through the induction of repairing chemical cascades in sequence. Evolutionary morphogenesis is a 4-dimensional creative process of which a temporal one: a $\Delta \mathrm{qV}$ of volume in a $\Delta \mathrm{qT}$ of four-dimensional time, guided by the membrane sensors, the cytoskeleton and the morphogen system. The realization of the biological project is the result of the actions on the molecular machines, of the internal and external chemical-physical information of the system, integrated, processed and translated by the genome and then realized through gene expression. The attractor to which the buffer system is addressed can be guided through simple or complex physical signals containing information that accelerate the kinetics of the molecular cascades. Any form of energy impacts a biological system by modifying the $\Delta \mathrm{G}^{\circ}$ and the functional dynamics of some multipotential signaling devices such as $\mathrm{Ca}^{2+}$, $\mathrm{G}$ protein coupled receptors, or the gene transcription system, is able to modify the activation or deactivation of molecular switches.

The cellular metabolism and function can be influenced from the outside of plasmamembranes by appropriate physical signals [1]. These can induce functional changes by piloting 
some important biochemical reactions [2] as well as the possibility of making the cells more resistant to hypoxia [3]. As demonstrated by the Piccardi effect, since the human body is made up of $80 \%$ of water, present in the cell as a polar solution, the magnetoelectric fields (MEF) are able to modify the intracellular chemical reactions [4]. MEFs are also able to modify the ionic and protonic flow of cell membranes, modifying the degree of polarization and permeability. MEFs modify cellular signaling by accelerating electronic transfer, through direct action on gap junctions and signal molecules, including morphogens [1]. All this is possible using complex electromagnetic field instruments, different from simple fields for field geometry, created through the production of harmonics by each portion of the wave. By sequencing a series of combinations of frequency, time, field geometry and signal intensity, an electrical information signal can be transferred to the reference target. The information must be sufficiently complex to activate simultaneously all the chemical cascades dedicated to the process to be obtained, starting from the formation of the fibrillar networks [5], passing through the ionic and protonic channels [6], continuing with short range and long range cell signaling. The fundamental characteristic of information is the possibility of being codified in a virtually infinite number of ways. The use of specific devices unites all the ways. The spoken words, for example, are constituted by fluctuations in the air pressure, those written by molecules of ink on paper, even the same thoughts depend on neural networks. The amount of information that can be carried by a radio signal is proportional to its frequency (modulation theorem). By modulation, means the transmission technique of an electric or electromagnetic signal, called modulating (possibly representing an information), by means of another electric or electromagnetic signal, called carrier, which has the purpose of converting the signal, from base band to translated band, according to the modulation theorem. The signals to be modulated can represent the most diverse information: audio, video, data. In general, the reason for using modulation is that the signals representing the information to be transmitted, are mostly of a low-pass nature (their spectral content is concentrated mostly at low frequencies), whereas the transmission channels, that more commonly are used to transmit more modulated signals at the same time (as hertzian channels and optical fibers) are of a band-pass nature. The spectrum of the electromagnetic signal representing the information must therefore be converted into a frequency. Moreover, the use of this technique allows to transmit electrical signals over long distances without overlapping other information. The carrier wave is a well-determined electromagnetic wave (much greater than the frequency of the modulating signal), which can be transmitted in air, in a vacuum, or by a suitable material means; for the experimentation a highly charged and conductive material represented by ionized silver was chosen. In general it is considered appropriate to use a modulated signal to reduce the effects of noise: the nature of the signal itself is such as to concentrate its spectrum in the lower frequencies, while transmission means have a better frequency response at higher frequencies. In this way can be avoid the distortion of the signal from the environment, so the device does not lose its effectiveness in the presence of any chemical / physical agent.

The electroweak theory presents a unified description of two of the four fundamental interactions of nature: electromagnetism and the weak nuclear force. Although these two forces seem very weak at the low energies of everyday life, the theory models them as two different aspects of the same force: above the unification energy, on the order of $10^{2} \mathrm{GeV}$, they become a single electrode-like interaction. The standard model (MS) is a theory that describes all the elementary particles known today and three of the four known fundamental forces, namely the strong interactions, the electromagnetic ones and the weak ones (the latter two unified in the ultra-weak interaction). This is a quantum field theory, consistent with both special relativity and quantum mechanics. To date, the MS forecasts have been largely verified experimentally with excellent accuracy. Endogenous magnetic fields are fluctuating ultra dry fields, derived from changes in the electric field given by variable dipoles, intra and extra cellular, in reaction to the dynamics of ionic flow and their interaction with polar proteins. They are continuously variable and can be analyzed with non-linear mathematical systems. Their non-linearity is closely linked to the non-linearity of the ionic flow. Given their intensity, in the order of the picoTesla, they can be influenced by external devices, with which they tend to create a balance [7]. This balance can be analyzed through stochastic physics. The endogenous fields are responsible, in part, together with the free charges of proteins and the activation states of the water molecules [8], of the electromagnetic "buoyancy" of the cytosol. (i.e. the ability of the sol to fluctuate the charged micro particles). This is also a physical characteristic of the cytosol which determines its relative density [9]. All this helps us to better understand how, since endogenous fields are so thin that they can be strongly conditioned, just an infinitesimal amount of energy (represented by the T-NES ${ }^{\circledR}-H I T$ Tech device) to adapt the cellular work to the conditions present moment by moment [10]. In light of the above, it is possible to state that the T-NES $\AA$-HIT Tech micronized silver patch device acts as a magnetoelectric vector complex (MEC), inducing a direct activation of proton and ionic fluxes from MEC, passing through glycocalyx, to the intracellular environment of the erythrocyte (optical - magneto electroacoustic path), activating the transmembrane ion channels. In this way it supplies sufficient energy to the phosphate groups of the ADP, not allowing excess lactic acid to be removed in the bloodstream, thus improving the muscular contraction work. The "endurance" training is very important because it allows you to increase and minimize the effort, which is to optimize and economize the technical gesture. The idea is to keep a given pace for a long time. The concept of "cruise speed", or "cruising speed", can be very useful. 


\section{Experimental Protocol}

\subsection{Animal Treatments}

Male BALB/C mice were purchased from IFFA Credo (L'Abreole, France). Mice were housed throughout the experiments in air-conditioned animal room and fed with a AIN-76 purified rodent diet composition (Dyets, Inc. Bethlehem, PA USA). All experimental protocols were carried out following the Guidelines for the Welfare of Animals in Experimental Research and the ECC Council Directive $86=609$, OJL 358, 1st December 1987. The tests were performed on 3 cohorts of "BALB / C" mice; each cohort was composed of 100 mice each, further subdivided into batteries of 10 different mice that were numbered. Overall, 300 mice were observed; each experiment was reiterated 3 times, therefore, in total, the final data are based on 900 surveys. The first cohort was treated with patches activated by micronized silver, the second cohort was treated with non-active patches while the third was left "untreated"

The patented device, T-NES ${ }$ - HIT Tech., (New Era Sport Technologies, Italy) is a disposable patch $(5 \mathrm{~cm} x 7 \mathrm{~cm})$, with an active central pad, based on micronized silver. The pad contains a nanotechnological material (silver micronized by plasma vaporization) that is appropriately treated with optical, magnetic, electrical and acoustic signals, in order to transfer very thin ELF magnetoelectric signals in the order of nanoTesla, such as to induce an action on cellular glycocalics, fundamental for cellular communication and recognition, by a direct activation of protonic and ionic fluxes and to guarantee a better transport of $\mathrm{O}_{2}$ in the blood cells; these signals also optimize the binding consistency in the musculoskeletal cells and lower the impedance in the nerve cells. This results in an increase in "endurance". All measurements were made in the oximeter chamber in which the rotarod system was inserted. Regarding the trace of the steps, it was used to verify the activity of each member of the cohort. The following parameters were measured: steady state generic training, long slow distance, high intensity "endurance" and steady state in split session training.

\subsection{Rotarod Test}

The rotarod test is one of the most commonly used tools for testing motor coordination and balance in mice because it is automated and does not require special training to administer the test. However, its sensitivity varies widely depending on the neuronal system (s) affected. This test requires mice to balance on a rotating rod, and their latency to fall is recorded as the endpoint measure. Mice can be tested at a set speed (usually the highest speed the mice can balance on) for different trials or at a gradually accelerated speed from 0 to 40 rpm over 5 min within a trial [11]

\subsection{Steady State Generic Training (SST)}

SST is an Aerobic training method that aims to maintain a constant heart rate, usually within moderate to medium-high intensity ranges, (i.e. between about 60 and $80 \%$ of the maximum heart rate [HR max], or between 50 and $75 \%$ of the maximum oxygen consumption). SST is very commonly performed on "Rotarod" machines, which allow to maintain some constant physiological parameters. The machines offer the possibility to optimize aerobic training through constant monitoring of heart rate. These devices allow you to set a specific difficulty or resistance so that it adapts to the range of the desired heart rate (internal load), relative to the specific training zones. In addition to providing the advantage of maintaining some constant physiological parameters, the performer is prevented from exceeding the anaerobic threshold, which is reached at medium-high intensity, and which could reduce the duration of the exercise. The SST on cardio machines further simplifies the organization of training as in addition to maintaining the constant heart rate, easier to monitor, requires little space to be performed, and uses only aerobic equipment. Although often only one machine is used (single machine mode), it is possible to alternate the execution of different circuit stations on different exercises during the training.

\subsection{Long Slow Distance or Low Intensity Steady State (LISST) Training}

More specifically, the lower SST intensities indicated for athletes would be between 70 and $80 \%$ of the VO2max, while for those wishing to improve or maintain well-being, levels between 50 and $60 \%-75 \%$ of the VO2max, or between 50 and $75 \%$ of the HRmax are appropriate. Low-intensity SST can be used for a variety of purposes, including improving the fitness of adults and the elderly, or during the early stages of aerobic training in different sports. LISST, or low-intensity SST, is probably the most popular and safe form of aerobic training, and for this reason it is suitable for non-athletes. LISST training became very popular since the sixties, but was introduced in the twenties by Dr. Ernst Van Auken, a German physiologist and trainer. In this case the main objective is distance or duration (potentially understood also as volume), rather than speed or intensity. LISST is less stressful at the cardiovascular and respiratory levels, but extreme distances can lead to joint wear and degeneration of muscle tissue and joints.

\subsection{High Intensity "Endurance" Training (HIET)}

High-intensity SST, around $85 \%$ of VO2max for athletes, and around $60 \%$ of $\mathrm{VO} 2 \mathrm{max}$ for non-athletes, is generally recommended for those who are already physically athletic. It is considered a useful training to improve cardiovascular and cardio-respiratory capacity, and aerobic power. Some authors define HIET as a Steady State performed between 85 and 95\% of the HRmax. For different athletes this intensity could underlie or exceed the anaerobic threshold. Scientific evidence has clearly shown that marathon runners run to a level that remains on the anaerobic threshold or very close to it. Research has revealed that in the context of aerobic activity, the intensity in which the energy derived from carbohydrates prevails over that derived from fats (called a crossover point) 
occurs at about $70-75 \%$ of the $\mathrm{VO} 2 \mathrm{max}$. However, recent evidence recognizes the benefits of high-intensity aerobic exercise (non-anaerobic intensities), suggesting that these levels are better suited to increasing aerobic fitness than moderate-to-moderate exercise.

\subsection{Steady State in Split Session Training (SSSST)}

There is another way in which the Steady State can be played, that is in split mode. This consists in suspending the performance for a certain period of time (10-60 minutes) and then repeating the exercise. For aerobic exercise divided in other terms is meant the performance of normal aerobic performance with constant trend (steady state training) of variable duration (15-60 minutes) divided by one or more rest periods of equally variable duration (10-60 minutes) ). For example, a divided aerobic session may consist of a steady state performance at moderate intensity for 20 minutes, followed by a total rest period for 20 minutes, then performing a similar 20-minute steady state performance again to the previous one. This process can be repeated more than once. The usefulness of this method can be found in a greater oxidation of fat. Stich et al. [12] analyzed the effects of two steady state performances conducted at $50 \%$ of the VO2max, both of which lasted 60 minutes, which were separated by 60 minutes of rest. They showed that the second between the two sessions favored a greater consumption of fat than the first. Goto et al. [13] demonstrated that two steady-state sessions of 30 minutes to $60 \%$ VO2max separated by 20 minutes of rest resulted in an increase in lipid expenditure compared to a single 60-minute steady state session performed at the same intensity. Goto et al. [14] confirmed the previous conclusions with three 10-minute aerobic efforts separated by 10 minutes of rest. These were able to lead to increased fat oxidation compared to 30 minutes of continued exercise.

\subsection{Analysis of the Step}

It is a not stressful test, but effective for evaluating the differences in the length of the step of the animals. Mice were induced to walk, non-forced, on a white absorbent sheet of paper, five times. Only walks that did not show hesitation or interruption were considered. If the animals stopped or there were episodes of grooming, the walks were discarded. Subsequently, the impressions left by the animals were analyzed, in particular the distance between two footprints of the same paw, or the stride length.

\subsection{Aerobic Capacity and Maximum Heart Rate}

The maximal oxygen transport capacity, during a protracted work over time is defined as aerobic capacity. The unit of measurement used is the VO2max, which is expressed in liters of oxygen ventilated in one minute, or in milliliters of oxygen per pound of body weight, ventilated in one minute. The maximum heart rate (HRmax) is the highest heart rate an individual can achieve without severe problems through exercise stress, and generally decreases with age. Since HRmax varies by individual, the most accurate way of measuring HRmax is via a cardiac stress test. In this test, a subject underwent to a controlled physiologic stress, while being monitored by an ECG. The intensity of exercise is periodically increased, until certain changes in heart function are detected on the ECG monitor, at which point the subject is directed to stop.

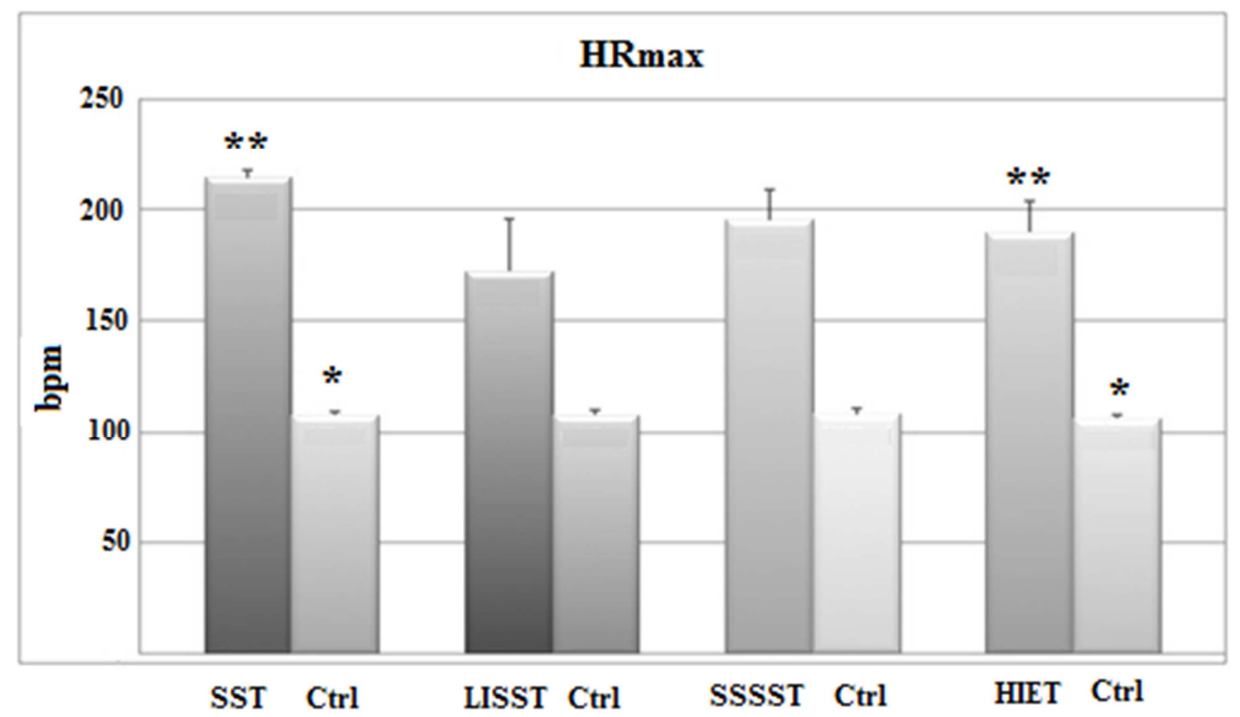

Figure 1. HRmax variation expressed as heart beats per minute (bpm) during SST, LISST, SSSST and HIET tests in treated-BALB / C mice compared to controls (Ctrl) as described in the Materials and Methods. Data represent the average of at least 100 observations $\pm S D$. Statistically significant $(P<0.05)$ increase of HRmax $(* *)$ reported in comparison to control $(*)(n=100)$.

\subsection{Maximum Aerobic Speed}

The Maximum Aerobic Speed (MVA) is the speed from which a human consumes the maximum possible amount of oxygen, thus reaches the threshold of maximum oxygen consumption. Beyond this threshold, most of the energy needed for the effort comes from aerobic respiration. Beyond 
the oxygen consumption remains constant and the extra power is supplied by lactic fermentation. The MVA is measured in $\mathrm{km} / \mathrm{h}$.

\subsection{Statistical Analyses}

The statistical analysis was performed using the Mann-Whitney U test for intergroup comparisons. Spearman's rank correlation test and the linear regression analysis were performed to analyze correlations between groups and the duration of tests. Values were expressed as the mean \pm SD. $P<0.05$ was regarded as statistically significant

\section{Results and Discussion}

In order to cope with the increased energetic demand and the increase of respiratory gases $\left(\mathrm{O}_{2}\right.$ and $\left.\mathrm{CO}_{2}\right)$ in the skeletal musculature, the execution of a physical exercise involves the functional integration of the respiratory, cardiovascular and muscular systems. The increase in metabolic demand, induced by the exercise, solicits these systems and therefore allows to evaluate their reserve capacity. Both the respiratory and the cardiovascular apparatus have a wide functional reserve and their possible alteration is clinically manifested only when this is greatly reduced. In fact, many of the methods, suitable for assessing the function of these rest units do not adequately reflect their reserve capacity; the reduction of this functional reserve, however, contributes significantly to the reduction of the exercise tolerance. The magnetic fields used for medical purposes, have shown that the interactions between the electromagnetic field (MEF) and human cells lead to changes in cellular metabolism. The individual components of a biological system immersed in a MEF undergo a stress due to the electrical charges present on molecules, ions and free radicals. While high-frequency MEFs can cause micro-vibrations due to resonance, cellular changes induced by alternating low-frequency fields are due to weak and slow inductions [15-16].

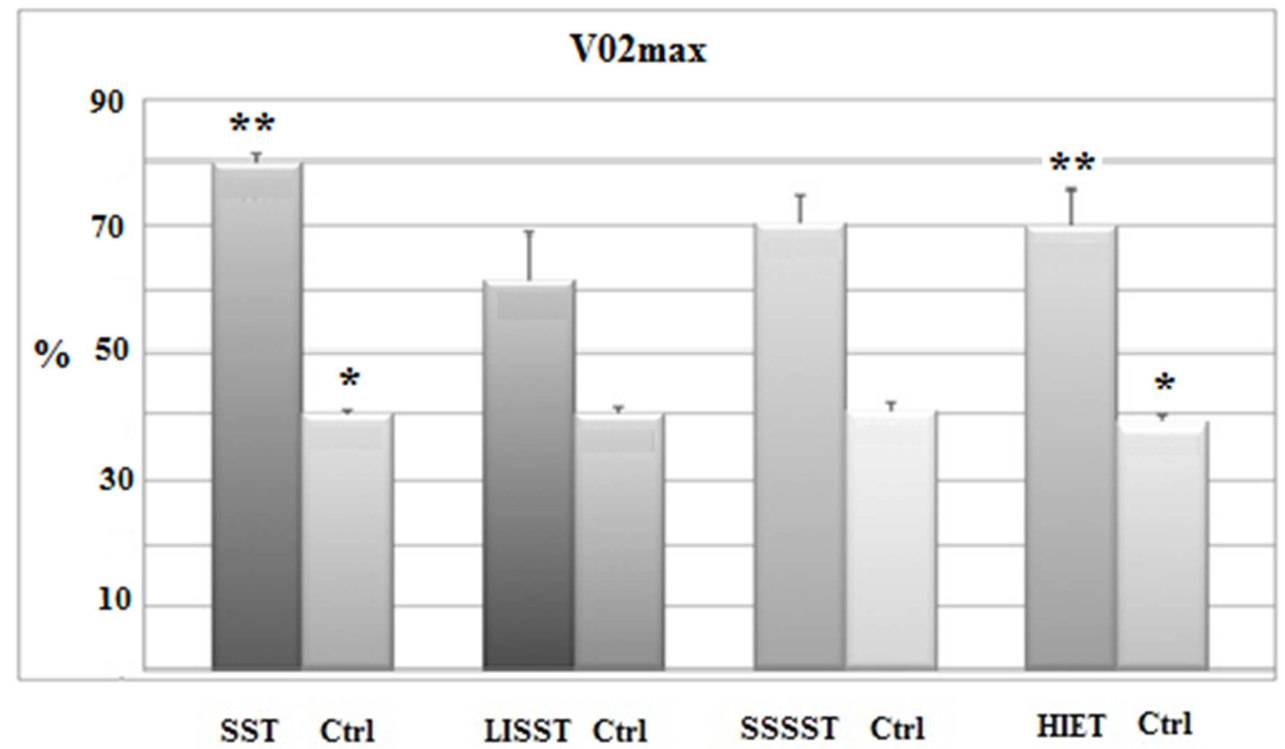

Figure 2. Percentage variation of V02max during SST, LISST, SSSST and HIET tests in treated-BALB / C mice compared to controls (Ctrl) as described in the Materials and Methods. Data represent the average of at least 100 observations $\pm S D$. Statistically significant $(P<0.05)$ increase of V02max (**) reported in comparison to control $(*)(n=100)$.

Since a complex biological system, like the cell, does not allow to predict which mechanisms are involved in its metabolism, the effect of these stresses is represented by the overall result. Previously reported experimental observations were made essentially "in vitro". These showed that MEF positively influences the metabolism of fibroblasts [17], chondrocytes [18] and osteoblasts [19], while modulating the effects of hormones and neurotransmitters on receptors of different cell types [20]. These fields are then able to modify some physiological parameters, such as proliferation, transcription, synthesis and secretion of growth factors [21-22] of the cells grown "in vitro". The aim of the present investigation was to verify if ELF fields influenced muscle stimulation in vivo, evaluating the potential improvement of "endurance" in "BALB / C" mice, treated with a nanotechnological patch device called T-NES®-HIT Tech.

The effect of the application of T-NES ${ }^{\circledR}-H I T$ Tech. micronized silver patch device on BALB / C mice, undergoing resistance training is presented as HRmax (Figure 1), V02 max (Figure 2) or MAV (Figure 3) in the various selected parameters. From data processing it is evident a similar increase in performance of about $98 \%$ for SST, $53 \%$ for LISST, $82 \%$ for SSSST and $79 \%$ for HIET, in all the evaluated training exercises. The endurance gained, by the T-NES ${ }^{\circ}$ HIT Tech patch system is to be considered included, in absolute value, between $30 \%$ and $35 \%$ compared to control animals. 


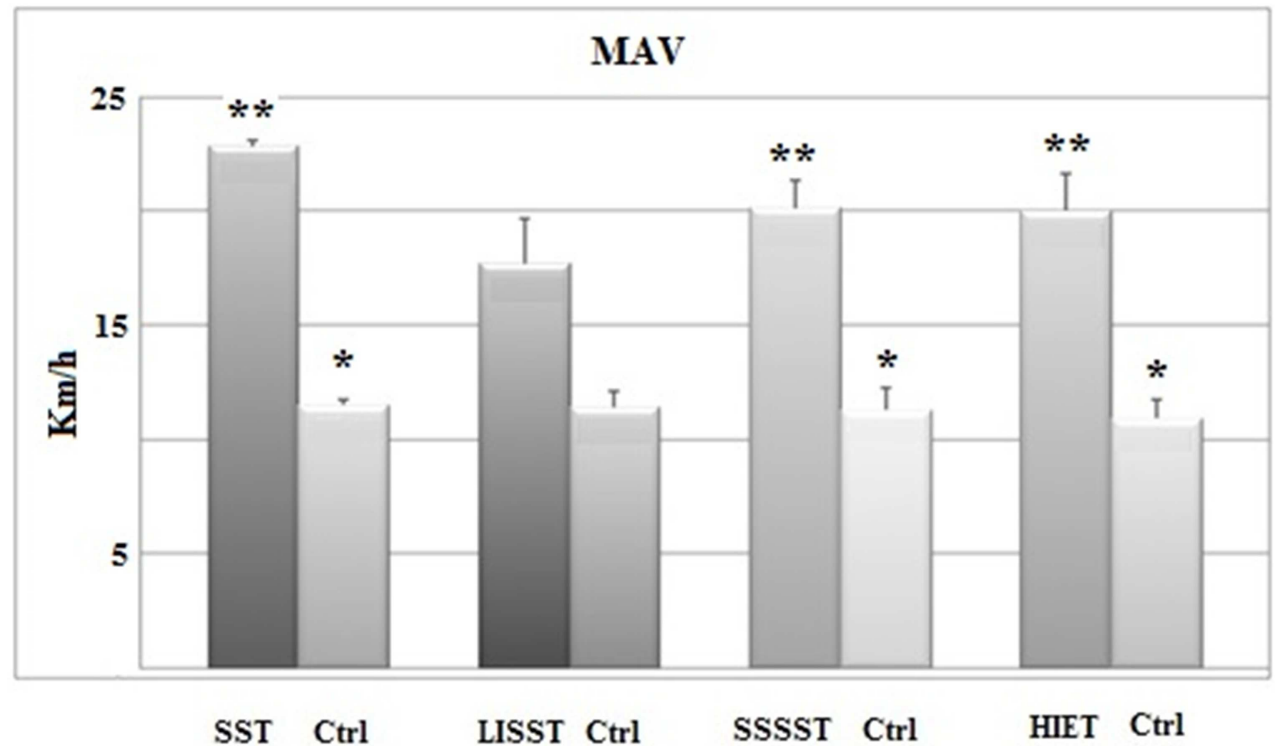

Figure 3. Percentage variation of MAV during SST, LISST, SSSST and HIET tests in treated-BALB / C mice, compared to controls (Ctrl) as described in the Materials and Methods. Data represent the average of at least 100 observations $\pm S D$. Statistically significant $(P<0.05)$ the increase of MAV (**) reported in comparison to control $(*)(n=100)$.

From the histological examination, carried out ex-post it can be defined that no animal has suffered: muscular physiopathological damages, muscular dystrophy, structural modification of the thyroid and physiological and/or physiopathological modifications of the cardiac musculature (data not shown).

\section{Conclusion}

The ELF magnetic field of a T-NES®-HIT Tech micronized silver patch device, applied to BALB/C mice, markedly improved their endurance performance as evidenced by the increase of their aerobic capacity, maximum heart rate and maximum aerobic speed, evaluated during various training Rotarod tests. This findings suggest that the application of the T-NES $\AA$-HIT Tech patch, could be extended to athletes to improve their sports performance.

\section{Acknowledgements}

We are grateful to B. S. and D. M. A. from University of Tor Vergata, Roma, Italy, for preparation and critical reading of the manuscript.

\section{References}

[1] F. Crescentini, "I Campi Magnetici Complessi come broadcasting di informazione bioattiva". MP\&BN Ed (2013).

[2] F. Crescentini, "La neo-osteomorfogenesi guidata con i CMF". Ed SIMPLE (2009).

[3] A. L. Di Carlo, J. M. Farrell, T. A. Litovitz. Myocardial protection conferred by electromagnetic fields. Circulation. 1999; 99 (6):813-816.

[4] G. B. Kauffman, L. Belloni. G. Piccardi (1895-1972), Italian physical chemist and master of the sun,. J. Chem. Educ., 1987; 64 (3): 205-208.

[5] B. Cuccurazzu, L. Leone, M. V. Podda, R. Piacentini, E. Riccardi, C. Ripoli, G. B Azzena, C. Grassi. Exposure to extremely low-frequency $(50 \mathrm{~Hz})$ electromagnetic fields enhances adult hippocampal neurogenesis in C57BL/6 mice. Exp Neurol. 2010; 226 (1):173-182.

[6] A. R. Liboff. Toward an electromagnetic paradigm for biology and medicine. J Altern Complement Med. 2004; 10 (1):41-47.

[7] Y. Zimmels. Thermodynamics in the presence of electromagnetic fields. Phys Rev E Stat Phys Plasmas Fluids Relat Interdiscip Topics. 1995; 52 (2):1452-1464.

[8] E. Del Giudice, S. Doglia, M. Milani. A Quantum Field Theoretical Approach to the Collectiv Behaviour of Biological Systems; Nuclear Physics. 1985; B251 (FS13):375-400.

[9] M. Namba, H. Watarai, and T. Takeuchi. Migration of Polystyrene Microparticles in Aqueous Media Caused by Electromagnetic Buoyancy. Analytical Sciences. 2000; 16 (1): 5-10.

[10] S. J. Williamson, L. Kaufman. Evolution of neuromagnetic topographic mapping. Brain Topogr. 1990; 3 (1):113-127.

[11] S. P. Brooks and S. B. Dunnett. Tests to assess motor phenotype in mice: a user's guide. Nat Rev Neurosci. 2009; 10 (7):519-529.

[12] V. Stich, I. de Glisezinski, M. Berlan, J. Bulow, J. Galitzky, I. Harant, H. Suljkovicova, M. Lafontan, D. Rivière, F. Crampes. Adipose tissue lipolysis is increased during a repeated bout of aerobic exercise. J. Appl. Physiol. 2000; 88 (4):1277-1283.

[13] K. Goto, N. Ishii, A. Mizuno, K. Takamatsu. Enhancement of fat metabolism by repeated bouts of moderate endurance exercise. J. Appl Physiol. 2007; 102 (6):2158-2164.

[14] K. Goto, K. Tanaka, N. Ishii, S. Uchida, K. Takamatsu. A single versus multiple bouts of moderate-intensity exercise for fat metabolism. Clinical Physiology Function Imaging. 2011; 31 (3):215-220. 
[15] G. Katsir, SC. Baram, AH. Parola. Effect of sinosoidally varing magnetic fields on cell proliferation and adenosine deaminase specific activity. Bioelectromagnetics. 1998; 1:46-52.

[16] P. Volpe. Interaction of zero-frequency and oscillating magnetic field biostructures and biosystems. Photochem Photobiol Sci. 2003; 2:673-648.

[17] J. C. Murray, R. W. Farndale. Modulation of collagen production in cultured fibroblasts by a low-frequency, pulsed magnetic field. Biochim Biophys Acta. 1985; 838:98-105.

[18] R. L. Smith, D. A. Nagel. Effects of pulsing electromagnetic fields on bone growth and articular cartilage. Clin Orthop Rel Res. 1993; 181:277-282
[19] C. T. Rubin, K. J. McLeod, L. E. Lanyon. Prevention of osteoporosis by pulsed electromagnetic fields. J. Bone Joint Surg Am. 1989; 71:411-417.

[20] W. R. Adey, J. C. Lin. Biological effects of radio frequency electromagnetic radiation. Electromagnetic Interaction with Biological Systems. Plenum 1999; 109-140.

[21] H. H. Guerkov, C. M. Lohmann, Y. Liu Y et al. Pulsed electromagnetic fields increase growth factor release by nonunion cells. Clin Orthop Rel Res. 2001; 384:265-279.

[22] S. M. Ross. Combined DC and ELF magnetic field can alter cell proliferation. Bioelectromagnetics. 1990; 11:27-36. 